\title{
Creencias del profesorado de Educación Física en Educación Primaria sobre la educación en valores
}

\section{Beliefs of Primary Education Physical Education teachers about education in values}

\author{
Marta Gutiérrez Sánchez ${ }^{1}$ \\ martags@um.es \\ Eduardo Romero Sánchez \\ eromero@um.es \\ TOMÁS IZQUIERDO RuS \\ tomasizq@um.es \\ Universidad de Murcia, España
}

\section{Resumen:}

Este trabajo tiene como propósito conocer las creencias del profesorado de Educación Física en Educación Primaria sobre la educación en valores. Desde un enfoque cualitativo se exploran las percepciones de 30 profesores de centros públicos de Educación Infantil y Primaria, de similar contexto socioeconómico, de la Región de Murcia, utilizando como instrumento de recogida de información la entrevista semiestructurada. Los resultados permitieron conocer aquellas variables que determinan la educación en valores así como vislumbrar algunos de los obstáculos que imposibilitan la educación integral del alumnado. Se concluye con algunas propuestas para avanzar hacia una educación que integre el componente ético y con líneas de investigación futuras sobre la temática.

\section{Palabras clave:}

Educación Primaria; Educación Física; Educación en valores; Profesorado.

\begin{abstract}
:
The purpose of this study is to examine the beliefs of Primary Education Physical Education teachers about education in values. From a qualitative perspective based on semi-structured interviews, the perceptions of 30 teachers from public Infant and Primary Education schools with a similar socioeconomic status in the Region of Murcia were explored. The results allowed for the determination of both the variables that define education in values and some of the obstacles that hinder the integral education of students. The study concludes with some proposals to advance towards a form of education that includes the moral component and with future lines of research in this area.
\end{abstract}

\section{Key words:}

Primary Education; Physical education; Education in values; teachers.

1 Dirección para correspondencia (correspondence address):

Marta Gutiérrez Sánchez. Universidad de Murcia. Facultad de Educación. Campus de Espinardo, s/n. 30100 Murcia (España). 
Creencias del profesorado de Educación Física en Educación Primaria sobre la

educación en valores

Marta Gutiérrez Sánchez, Eduardo Romero Sánchez y Tomás Izquierdo Rus

\section{Résumé:}

Ce travail a pour objectif de connaître les croyances du professorat d'Education Physique en Enseignement Primaire sur l'éducation dans des valeurs. D'un point de vue qualitatif, on explore les perceptions de 30 professeurs de centres publics d'Education Infantile et Primaire, dans un contexte socio-économique similaire, de la Région de Murcie, en utilisant, comme instrument de collecte d'information, I'interview semi structurée. Les résultats ont permis de découvrir les variables qui déterminent l'éducation dans des valeurs ainsi que reconnaître certains des obstacles qui empêchent l'éducation intégrale de l'ensemble des élèves. Nous concluons avec quelques propositions pour avancer vers une éducation qui intègre le composant moral et avec de futures lignes de recherche sur la thématique.

\section{Mots clés:}

Education Primaire; Education Physique; éducation aux valeurs; professorat.

Fecha de recepción: 13-3-2018

Fecha de aceptación: 9-5-2018

\section{Introducción}

Aunque siempre ha sido así, la sociedad del siglo XXI exige, aún más, la presencia de valores en la educación. La crisis económica y financiera está ocasionando cada vez más desigualdades y la explotación sin descanso de los recursos naturales está dando lugar a una destrucción sin precedentes del medio ambiente. Por otro lado, la presencia de multitud de culturas y subculturas en el escenario social está provocando enfrentamientos y una mirada al "otro" como extraño y no como portador de otros puntos de vista que contribuirían al progreso de la sociedad.

Como afirma Ruiz (2010), retomando a intelectuales de la corriente posmoderna como Bauman, Vattimo o Lyotard entre otros, nos encontramos en un momento en que la confianza ciega en la razón de la modernidad, que posibilitaba referentes de verdad para ordenar la actividad social y humana, se ha sustituido por "una multiplicidad de perspectivas, la fragmentación del pensamiento y una amplia gama de enfoques y vo-

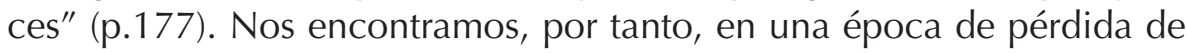
referentes morales que la escuela debe contribuir a rescatar prestando especial atención a la diversidad cultural, religiosa e ideológica.

Los teóricos de la posmodernidad nos indican que las vidas de las personas han dejado de sentirse aprobadas o legitimadas por algún criterio unificador.

Es como si al perder los referentes unificadores se produjera una especie de 
fragmentación en nuestra vida que nos conduce a proceder con criterios dispares, e incluso a veces contradictorios, en cada uno de nuestros ámbitos (Raventós y Prats, 2012, p. 21).

En este sentido, el diálogo, la cooperación, la solidaridad y la tolerancia son valores que la escuela ha de incluir tanto en sus presupuestos teóricos y legislativos como en los prácticos para contrarrestar los valores economicistas y meramente estéticos que priman actualmente en la sociedad y que hacen de la competitividad, el consumismo y el individualismo sus señas de identidad. Es necesario que pensemos todos los ámbitos del sistema educativo desde presupuestos que permitan que el alumnado se enfrente al escenario social de la actualidad. Para ello, no se puede entender una educación sin su esencia misma, sin su componente axiológico.

\section{La dimensión ética de la educación}

Aunque existen diferentes presupuestos, concepciones y sentidos de la educación, sobre todo en su relación con la dimensión axiológica, ética y ciudadana, hoy, ya parece indiscutible considerarla, como algo más que pura instrucción o formación en sentido academicista o instrumental (Reboul, 2009). Por este motivo la cuestión de los valores, y el entramado ético que sustenta una determinada concepción de la educación, merece especial atención.

No existe educación sin que de manera explícita o implícita estén presentes los valores. Por tanto, la dimensión ética no es un añadido sino una parte constitutiva de la misma. De no ser así, se podría hablar de aprendizaje, pero en ningún caso, estaríamos ante acciones verdaderamente educativas (Ortega y Mínguez, 2001).

Esta cuestión ha quedado reflejada en los diversos planteamientos legislativos desde los años ochenta hasta la actualidad. La inclusión de los valores en las finalidades educativas ha sido una constante en el Sistema Educativo Español, aunque sus planteamientos epistemológicos y terminológicos hayan diferido en las diversas leyes educativas que han estado vigentes en las últimas décadas.

La misma LODE (Ley Orgánica del Derecho a la Educación de 1985) ya ensayó esta incorporación dentro del área de Convivencia en la segunda etapa de Educación General Básica (EGB), en el área de Ciencias 
Sociales, en la asignatura de Ética como alternativa a la clase de Religión del Bachillerato Unificado Polivalente (BUP) e, incluso, a través de una frustrada enseñanza obligatoria de la Constitución también en el bachiIlerato (Gómez Rodríguez, 2008), sobre todo desde el presupuesto de que tal materia atendía a una necesidad defendida en numerosos foros internacionales y sistemas educativos, en respuesta a situaciones complejas generadas por la misma democracia.

Sin embargo, fue en 1990, con la llegada de la LOGSE (Ley de Ordenación General del Sistema Educativo), cuando se apostó de forma más clara y decidida por la incorporación de la educación en valores éticos y cívicos, de modo transversal, es decir, a través del currículo de las diferentes áreas. El objetivo último era formar en el compromiso democrático a los jóvenes, en respuesta a los retos de una sociedad compleja que requería reactivar la respuesta ética y política ante asuntos como la globalización y la multiculturalidad, la tecnología de la comunicación y la información, la violencia, la falta de sentido de lo público y lo común, la promoción de una conciencia ciudadana y participativa o la necesidad de cohesión social (Altarejos y Naval, 2005; Bolívar, 2007; Gracia y Gozálvez, 2016).

Sin embargo, el loable intento de tratar de incorporar los valores cívicos y éticos a través de los llamados temas transversales no dio los resultados esperados, ocurriendo con frecuencia que estos se veían trasladados a un plano marginal y perfectamente eludible al no tener que ser explícitamente evaluados como sucedía con el resto de las áreas disciplinares.

Ante esta inoperancia de la transversalidad, la Ley Orgánica de Educación (LOE) de 2006 incorporó de manera decidida y específica la educación en valores cívicos ("Educación para la ciudadanía") como materia obligatoria y autónoma curricularmente, presente tanto en Educación Primaria como en Educación Secundaria Obligatoria y Bachillerato.

La llegada de la LOMCE (Ley Orgánica para la Mejora de la Calidad Educativa) supone un declarado cambio respecto de la LOE en relación al tratamiento educativo de los valores éticos y ciudadanos. En primer lugar, y prestando atención a la Educación Primaria a lo largo de los seis cursos que la componen, el alumnado ha de ir decantándose entre educación religiosa y educación ética. De esta forma, los valores éticos quedan reducidos a la optatividad lo que significa que tal materia no ha de ser cursada por todo el alumnado. 
Sin embargo, contradictoriamente la LOMCE establece en el título XIV lo siguiente:

Uno de los principios en los que se inspira el Sistema Educativo Español es la transmisión y puesta en práctica de valores que favorezcan la libertad personal, la responsabilidad, la ciudadanía democrática, la solidaridad, la tolerancia, la igualdad, el respeto y la justicia, así como que ayuden a superar cualquier tipo de discriminación (p. 97866).

Así mismo, otorga especial importancia a planteamientos metodológicos que aboguen por la incorporación de forma transversal de la educación cívica en todas las asignaturas durante la educación básica.

Esta última cuestión queda patente en una organización curricular orientada a la adquisición de competencias básicas que implican asumir que existe un componente ético que se ha de planificar y evaluar del mismo modo que se hace con el cognitivo y con las habilidades.

En la Orden ECD/65/2015, de 21 de enero, se establece con rotundidad los fines educativos vinculados con las competencias consideradas básicas para las diferentes etapas educativas. En este documento aparecen veinticinco referencias a los valores relacionados con las diferentes competencias, de cara a la formación integral de la persona tanto en la educación formal (etapas de Educación Primaria, Secundaria y Bachillerato), como en la educación no formal e informal (Gracia y Gozálvez, 2016).

\section{Los valores en la Educación Física}

Teniendo en cuenta estos planteamientos, la asignatura de Educación Física en la etapa de Educación Primaria ha de contribuir a la formación cívica de los escolares de la misma manera que lo hacen el resto de las asignaturas porque, en definitiva, como afirman Ruiz y Cabrera (2004), la "educación física es ante todo educación" (p.11) y, de este modo, los profesores del área deben contribuir a la promoción y el desarrollo de valores.

De hecho, si nos detenemos en su actual planteamiento curricular en la etapa de Educación Primaria, la Educación Física aparece como un medio privilegiado para el desarrollo de actitudes éticas. Específicamente, se plantea como una asignatura que ha de articularse desde el punto 
de vista curricular en torno a cinco situaciones motrices diferentes entre las que destacan, entre otras, las acciones motrices en situaciones de cooperación. Por otro lado, en todos los bloques de contenidos se insiste en la necesidad de incorporar valores sociales e individuales.

No cabe duda de que la Educación Física es un área que por su naturaleza sitúa la educación en valores en un lugar privilegiado (Marín, 2013; Pascual et al., 2011; Prat, 2001; Prat, Font, Soler y Calvo, 2004; Wrigth y Burton, 2008). La superación, la autonomía, la cooperación y la responsabilidad son valores que suelen estar presentes en las clases de Educación Física al generar situaciones que favorecen la interacción y las relaciones con los demás (Gutiérrez, 2003; Hellison, 2011; Moncada, 2005).

Pero para que la Educación Física contribuya de manera decidida al desarrollo ético de los escolares es necesario que se consideren algunos factores. Si atendemos a lo que sugiere la literatura especializada en esta línea, la inclusión de los valores en los diseños curriculares y en el Proyecto Educativo de Centro, variables relacionadas con la práctica docente y la cultura organizativa y moral de los centros de enseñanza son factores decisivos para que tenga lugar la educación en valores.

Así, y en relación con aspectos curriculares se ha señalado la importancia de su presencia en el currículum (Gómez, 2003; Gutiérrez, 2003; Pavesio y Rivera, 2001), y la necesidad de planificarlos e incluirlos en las programaciones docentes puesto que la práctica deportiva no asegura por sí sola la educación en valores (Sánchez, 2002; Venero, 2007).

Asimismo, se ha insistido en la importancia de su institucionalización a través de su presencia en los planes y proyectos que se desarrollan en los centros, de manera que se asegure el compromiso de todos los miembros de la comunidad escolar (Prat, 2001; Prat y Soler, 2003).

Por otro lado, aspectos vinculados a la práctica docente constan en la literatura especializada como elementos a considerar para que tenga lugar una educación que incluya el componente ético. Se señalan aquellos directamente relacionados con el profesorado, insistiendo en las relaciones que éstos establecen con el alumnado, las demostraciones que realiza, la forma de informar y comunicarse y el control del grupo, y factores de carácter organizativo que tienen que ver con el uso de espacios y materiales y con la formación de los grupos (Gómez Rijo, 2005; Prat, 2001; Prat y Soler, 2003). Por tanto, para que tenga lugar una educación en valores en las clases de Educación Física será 
crucial la manera en la que el profesorado organice e imparta la sesión (Evans, 2001).

La cultura organizativa del centro constituye también un referente clave para la educación en valores. Las relaciones que se establezcan en el centro, las formas de organización, los códigos de conducta, etc. determinan que se genere una atmósfera adecuada que posibilite la educación en valores (Bolívar, 2007).

Del mismo modo, es evidente que la colaboración de la familia es crucial para el desarrollo ético de los escolares puesto que es tanto en el ámbito escolar como en el familiar donde se adquieren valores. Así, la literatura pedagógica tanto de carácter general como la directamente vinculada a la Educación Física en Educación Primaria pone de manifiesto la importancia de la implicación de la familia para la educar en valores (Arufe, 2011; Ortega y Mínguez, 2001;Peiró, 2013).

En definitiva, la educación en valores requiere una intencionalidad educativa, una integración curricular y organizativa que se plasme en un proyecto institucional compartido por todos los miembros de la comunidad escolar. La escuela, como organización, ha de definir los valores en los que posicionarse (Venero, 2007) e incluirlos en sus planteamientos organizativos y curriculares para que tenga lugar una educación integral del alumnado.

Precisamente por la complejidad que subyace articular todos los elementos que determinan la educación en valores, existen algunas dificultades vinculadas a los mismos, necesarias de identificar para contribuir a una educación acorde con las exigencias a las que tiene que atender la escuela en la actualidad. En este sentido, se han identificado dificultades relacionadas con la formación del profesorado, la inexistencia de un consenso explícito sobre los fines de la educación, la escasa implicación de las familias o la inexistencia de proyectos de centro en los que se asuma el trabajo en valores. Así, Agúndez (2015) ha manifestado que uno de los problemas para educar en valores reside en la falta de un consenso por parte de la sociedad sobre los valores en los que educar, Santos Guerra (2010) ha insistido en la escasa formación del profesorado y en el valor que se le otorga a enseñar materias frente al compromiso que han de tener los docentes para educar en principios éticos, y Martínez, Esteban y Buxarrais (2011) han aludido a la escasa formación de las familias afirmando que "no todos los padres y madres son igualmente competentes para ejercer como auténticos educadores morales" (p.98). 


\section{Método}

En este trabajo se ha optado por un enfoque metodológico cualitativo, ya que esta metodología es apropiada para establecer conexiones entre la práctica educativa y los significados que le otorgan los actores que participan en ella y, en consecuencia, el más idóneo para abordar los objetivos del trabajo. Optar por una metodología cualitativa nos permite acercarnos al escenario natural y convertir éste en la fuente directa de los datos que se obtienen (Colás, Buendía, y Hernández, 2009). Por ello, se considera la entrevista cualitativa como la técnica más adecuada para este estudio ya que, según Buendía, Colás y Hernández (1998), permite obtener información sobre lo que es importante y significativo para los informantes $y$, en consecuencia, resulta fundamental para comprender su propia visión del mundo.

\section{Objetivos}

A pesar de contar con amplia y fecunda literatura especializada que identifica tanto los factores que determinan la educación en valores como los problemas, son inexistentes los estudios que se han preocupado en exclusividad por conocer las creencias del profesorado de Educación Física en relación a estas cuestiones.

Constatada la escasez de investigaciones realizadas en nuestro contexto, y teniendo en cuenta la literatura especializada, los objetivos principales de este trabajo fueron conocer las percepciones del profesorado de Educación Física de la etapa de Educación Primaria sobre las dificultades para educar en valores e indagar acerca de sus percepciones sobre los factores que determinan la adquisición de actitudes y valores éticos por parte del alumnado. De este último objetivo, y en coherencia con lo que sugieren los planteamientos pedagógicos plasmados en este trabajo, se desprendieron tres específicos:

- Conocer las percepciones de los profesores de Educación Física en Educación Primaria sobre la finalidad del área y sobre qué valores han de articular la propuesta pedagógica.

- Conocer la percepción del profesorado sobre la inclusión de los valores en los distintos niveles de concreción curricular y sobre cómo ha de ser la trama organizativa de centro para la educación en valores. 
- Conocer las percepciones de los profesionales del área de Educación Física sobre cómo ha de ser la práctica docente para favorecer el desarrollo ético de los escolares.

Conocer las creencias del profesorado sobre estas cuestiones es de utilidad para vislumbrar el estado de la educación en valores en la actualidad en los centros de Educación Primaria. Indagar sobre esta cuestión podría constituir el punto de partida para el establecimiento de propuestas pedagógicas que posibilitarán avanzar hacia una intervención educativa y orientación institucional que posibilite una educación más humana.

\section{Participantes}

Los participantes de esta investigación son profesores y profesoras de Educación Física en Primaria de centros públicos de la Región de Murcia de similar contexto socioeconómico. La selección de los participantes estuvo guiada fundamentalmente por criterios que aseguraran que la información recogida sería relevante para los objetivos de la investigación. De este modo, los criterios de selección de los participantes se relacionaron con indicadores que aseguraran que los participantes tuvieran un conocimiento profundo y global de variables de tipo pedagógico, curricular y organizativo. Concretamente, los criterios utilizados para la selección de los participantes fueron los siguientes:

1. Tener, al menos, cuatro años de experiencia como maestro de Educación Física en la etapa de Educación Primaria en el mismo centro educativo.

2. Haber sido tutor/a de un grupo de alumnos/as, al menos en dos ocasiones.

3. Ser responsable de planes o proyectos de centro vinculados al fomento de la actividad física y el deporte, coordinador/a pedagógico o miembro del equipo directivo.

Los participantes seleccionados, teniendo en cuenta los criterios mencionados, fueron 10 mujeres y 20 hombres. Todos ellos han sido tutores/as de un grupo, al menos en dos ocasiones, y el 34\% de los mismos ostenta actualmente esa responsabilidad. Del total de los participantes el $26.6 \%$ son miembros del equipo directivo, el $20 \%$ responsables del Plan de Educación para la Salud en la Escuela, el 33.3\% responsables de Deporte Escolar y el 20\% coordinadores de tramo. 
Creencias del profesorado de Educación Física en Educación Primaria sobre la educación en valores

Marta Gutiérrez Sánchez, Eduardo Romero Sánchez y Tomás IzQuierdo Rus

\section{Instrumento}

Se utilizó la entrevista semiestructurada como técnica de recogida de información. La elaboración del guion de la entrevista se basó en el establecimiento de cinco tópicos o dimensiones basados en los constructos de la fundamentación teórica y en los objetivos de la investigación. Se delimitaron cinco áreas en las que se introdujeron una serie de preguntas lo que permitió acotar la información evitando su dispersión. Concretamente, se consideraron inicialmente las siguientes dimensiones: naturaleza del área, organización curricular, práctica docente, cultura organizativa y dificultades para educar en valores.

En la primera dimensión se incluyeron preguntas para recoger información sobre las percepciones de los profesionales sobre los fines y la potencialidad del área para educar en valores. En la segunda, cuestiones vinculadas a la concreción de los valores en el currículum actual y en su presencia en el Proyecto Educativo de Centro y en las programaciones docentes. En la dimensión práctica docente, se incluyeron preguntas que pretendían indagar acerca de la percepción de los profesionales sobre cómo han de ser los procesos de transmisión de la información para contribuir a la educación en valores, los agrupamientos del alumnado y los recursos. En relación al tópico cultura organizativa, las preguntas iban dirigidas a indagar sobre cómo influyen las relaciones que se generan en la comunidad escolar en la educación en valores.

Se llevó a cabo una revisión por parte de tres jueces externos, todos ellos expertos en educación en valores y con una amplia trayectoria investigadora en esta temática. Tras la revisión por parte de los expertos se Ilevaron a cabo algunas reestructuraciones de las dimensiones y de las preguntas asociadas a cada una de ellas. El tópico denominado naturaleza del área se subdividió en dos, diferenciándose así entre fines de la Educación Física y naturaleza del área. En lo relativo a la organización curricular, se añadió una pregunta para recoger información acerca de las percepciones de los profesionales sobre la contribución a la educación en valores de los planes y proyectos, incluidos en la Programación General Anual, que se desarrollan en el centro. En la dimensión práctica docente se sustituye la pregunta relacionada con cómo han de ser los procesos de transmisión de la información para educar en valores por otra más genérica que hace alusión a cómo ha de ser la relación educativa para contribuir al desarrollo ético de los escolares. En cuanto a la 
dimensión cultura organizativa, los jueces coincidieron en la idoneidad del planteamiento inicial de las preguntas.

Por último, se incluye un nuevo tópico, denominado consenso ético, con el objetivo de indagar sobre cuáles son aquellos valores que la escuela ha de contribuir a desarrollar.

Posteriormente, se realizó una prueba piloto con un profesor de Educación Física que imparte docencia en Educación Primaria y realiza tareas docentes e investigadoras de manera paralela en el ámbito universitario, constatándose la idoneidad del instrumento para dar respuesta a los objetivos de la investigación. (Tabla1)

A continuación, se describe detalladamente cada una de las categorías:

Fines: Se quería profundizar sobre el concepto que tiene el profesorado acerca de la educación y su vinculación necesaria con los valores. Esta categoría se asienta en una concepción pedagógica que entiende que los valores son ineludibles para el desarrollo social e individual. Está ligada al ideal de hombre y de sociedad que se pretende transmitir a través de la acción educativa asumiendo que educar es una tarea moral. Supone reconocer que necesariamente la tarea educativa se lleva a cabo con una referencia normativa que acude a un conjunto de valores más o menos consciente.

Consenso ético: Se pretendía conocer si existe un acuerdo entre los valores que hay que contribuir a desarrollar en la etapa de Educación Primaria. Se trata de avanzar en la definición de una base moral en la que ha de apoyarse la acción educativa. Perseguía responder a la pregunta en qué valores educar. Potencialidad: Se persigue indagar sobre la potencialidad del área de Educación Física para educar en valores. La educación física ha sido considerada como un lugar privilegiado para el desarrollo de valores sociales y personales en la medida en que la naturaleza del área obliga al niño a aprender a competir, resolver problemas, dialogar, superarse, ganar, perder... (Ruiz y Cabrera, 2004).

Currículum: El objetivo es recoger información sobre la percepción del profesorado acerca de la inclusión de los valores en el currículum actual. Se parte de que el currículum recoge aspiraciones e ideas y una concepción de la inclusión y tratamiento de los valores. El currículum está marcado por una determinada orientación educativa de corte más instructivo o tecnológico o de corte más educativo o axiológico.

Consenso explícito a nivel de centro: Se pretendía indagar sobre cómo perciben los profesionales el consenso que existe a nivel de centro sobre la 
transmisión de la dimensión actitudinal y ética. Concretamente en cómo se reflejan las pretensiones legislativas en las finalidades educativas que constan en el Proyecto Educativo de Centro, en la organización y funcionamiento del centro y en los planes y proyectos que se desarrollan en la institución escolar. Desarrollo curricular: Se pretendía averiguar la percepción del profesorado sobre la presencia de los valores en el desarrollo curricular del área. Concretamente, en cómo introduce el profesorado, coherente y articuladamente, las actitudes y valores en la programación docente del área. Se parte de la concepción de que en las programaciones docentes no sólo se incluyen contenidos que tiendan al desarrollo de competencias motrices sino también a la adquisición de actitudes y valores.

Práctica docente: Se pretendía indagar sobre cómo entienden los profesionales que han de organizarse las variables que intervienen en el proceso de enseñanza y aprendizaje para educar en valores. Se parte de la concepción de que las actitudes y los valores están implícitas en la manera en que se transmiten los contenidos y en las relaciones que se establecen en el aula. Se asume que los valores se viven y que, por tanto son fruto de la experiencia. Clima ético del centro: Se perseguía indagar sobre la percepción de los profesionales acerca de cómo ha de ser el clima organizativo del centro escolar para favorecer la educación en valores. Partimos de que en función de cómo está configurado organizativamente un centro, la educación en valores cobrará un sentido u otro. Asumimos que la educación en valores implica un clima relacional de colaboración entre el profesorado y una visión común de la función de la institución escolar entre familias y profesorado.

Problemas: Se pretendía dar cuenta del conjunto de problemas que afectan a la educación en valores según la visión de los/as profesionales.

Tabla 1

Instrumento de recogida de información

\begin{tabular}{|c|c|}
\hline CATEGORÍA & PREGUNTAS \\
\hline FINES & ¿Cuál es la finalidad de la Educación Física? \\
\hline $\begin{array}{l}\text { CONSENSO } \\
\text { ÉTICO }\end{array}$ & $\begin{array}{l}\text { ¿Cuáles son los valores que de manera más urgente he- } \\
\text { mos de trabajar en Educación Primaria? }\end{array}$ \\
\hline POTENCIALIDAD & $\begin{array}{l}\text { ¿En qué medida contribuye el área de Educación Física a } \\
\text { la educación en valores? } \\
\text { ¿Cuáles son los valores que se trabajan en mayor medida } \\
\text { en el área de Educación Física? }\end{array}$ \\
\hline
\end{tabular}




\begin{tabular}{|c|c|}
\hline CATEGORÍA & PREGUNTAS \\
\hline CURRÍCULUM & $\begin{array}{l}\text { ¿En qué medida contribuye el currículum de Educación } \\
\text { Física a la educación en valores? }\end{array}$ \\
\hline CONSENSO & ¿En qué medida el Proyecto Educativo contribuye a la \\
\hline EXPLÍCITO & educación en valores? \\
\hline A NIVEL DE & ¿En qué medida los planes, proyectos y experiencias de \\
\hline CENTRO & centro contribuyen a la educación en valores? \\
\hline DESARROLLO & ¿Cuál es su percepción acerca de la presencia de los valo- \\
\hline CURRICULAR & res en las programaciones docentes de Educación Física? \\
\hline PRÁCTICA & ¿Cómo considera que hade ser la relación educativa para \\
\hline \multirow[t]{2}{*}{ DOCENTE } & educar en valores? \\
\hline & $\begin{array}{l}\text { ¿Considera que los agrupamientos determinan la educa- } \\
\text { ción en valores?; ¿cómo considera que han ser los agru- } \\
\text { pamientos para favorecer la educación en valores? } \\
\text { ¿Cuáles considera que son los recursos (instalaciones, } \\
\text { espacios, materiales) más apropiados para la educación } \\
\text { en valores? }\end{array}$ \\
\hline CLIMA ÉTICO & ¿Cómo considera que influyen las relaciones que se esta- \\
\hline \multirow[t]{4}{*}{ DEL CENTRO } & $\begin{array}{l}\text { blecen en la comunidad escolar para el desarrollo ético } \\
\text { de los escolares? }\end{array}$ \\
\hline & $\begin{array}{l}\text { ¿Cómo afecta la relación con la familia en el desarrollo } \\
\text { ético de los escolares? }\end{array}$ \\
\hline & $\begin{array}{l}\text { ¿Es importante la coordinación del profesorado para la } \\
\text { educación del alumnado?; ¿cómo se coordinan en este } \\
\text { sentido? }\end{array}$ \\
\hline & $\begin{array}{l}\text { ¿Se coordina con otros especialistas (Orientadora, traba- } \\
\text { jadora social de barrio, asociaciones...) para facilitar el } \\
\text { aprendizaje de los contenidos éticos? }\end{array}$ \\
\hline PROBLEMAS & $\begin{array}{l}\text { ¿Qué dificultades cree que existen para educar en valo- } \\
\text { res? }\end{array}$ \\
\hline
\end{tabular}

\section{Procedimiento}

Se contactó telefónicamente con uno de los miembros del equipo directivo de los centros indicándoles el objetivo de la investigación y los criterios de selección de los participantes. Teniendo en cuenta los criterios antes mencionados, los equipos directivos se pusieron en contacto con el profesorado especialista en Educación Física, les describieron el objetivo de la investigación y se aseguraron de que tenían voluntad por realizar las entrevistas. Posteriormente, se contactó nuevamente vía telefónica con el equipo directivo y se fijó la fecha y la hora de realización de las entrevistas. 
Las entrevistas se llevaron a cabo en los centros donde imparten docencia el profesorado participantes durante una de sus horas complementarias y en aulas que garantizaron la inexistencia de interrupciones.

Antes de la realización de las entrevistas se les informó a los participantes del propósito del estudio y se les garantizó el anonimato, así como el uso confidencial de la información obtenida. La realización de las entrevistas tuvo un tiempo promedio de treinta minutos.

\section{Resultados}

Esta investigación pretende aportar una descripción de las percepciones del profesorado de Educación Física en Educación Primaria sobre los factores que determinan la adquisición de actitudes éticas de los escolares y sobre las dificultades que existen para educar en valores. Estas percepciones han quedado plasmadas a través de redes semánticas donde se muestran las diferentes categorías analizadas.

En la figura 1 se reflejan las tres primeras categorías (fines, consenso ético, potencialidad del área) ya descritas anteriormente en este estudio. En relación con la finalidad del área, los entrevistados manifiestan que una de las prioridades de la Educación Física en Educación Primaria es el desarrollo de valores. Aluden también a la importancia de contribuir al desarrollo motriz y a la adquisición de hábitos para la práctica de actividad física, pero sitúan el componente ético en un lugar privilegiado.

En cuanto a la categoría consenso ético, los resultados del estudio advierten que el profesorado comparte la necesidad de educar en unos determinados valores. El respeto, la solidaridad, la colaboración y la responsabilidad son valores que el profesorado de Educación Física en Primaria considera urgentes de desarrollar.

Por otro lado, el profesorado de Educación Física en Educación Primaria percibe el área como un espacio privilegiado para educar en valores porque su carácter procedimental favorece a que se den aquellas relaciones afectivo-sociales que obligan a la resolución de conflictos y al establecimiento de unas relaciones sociales positivas. 


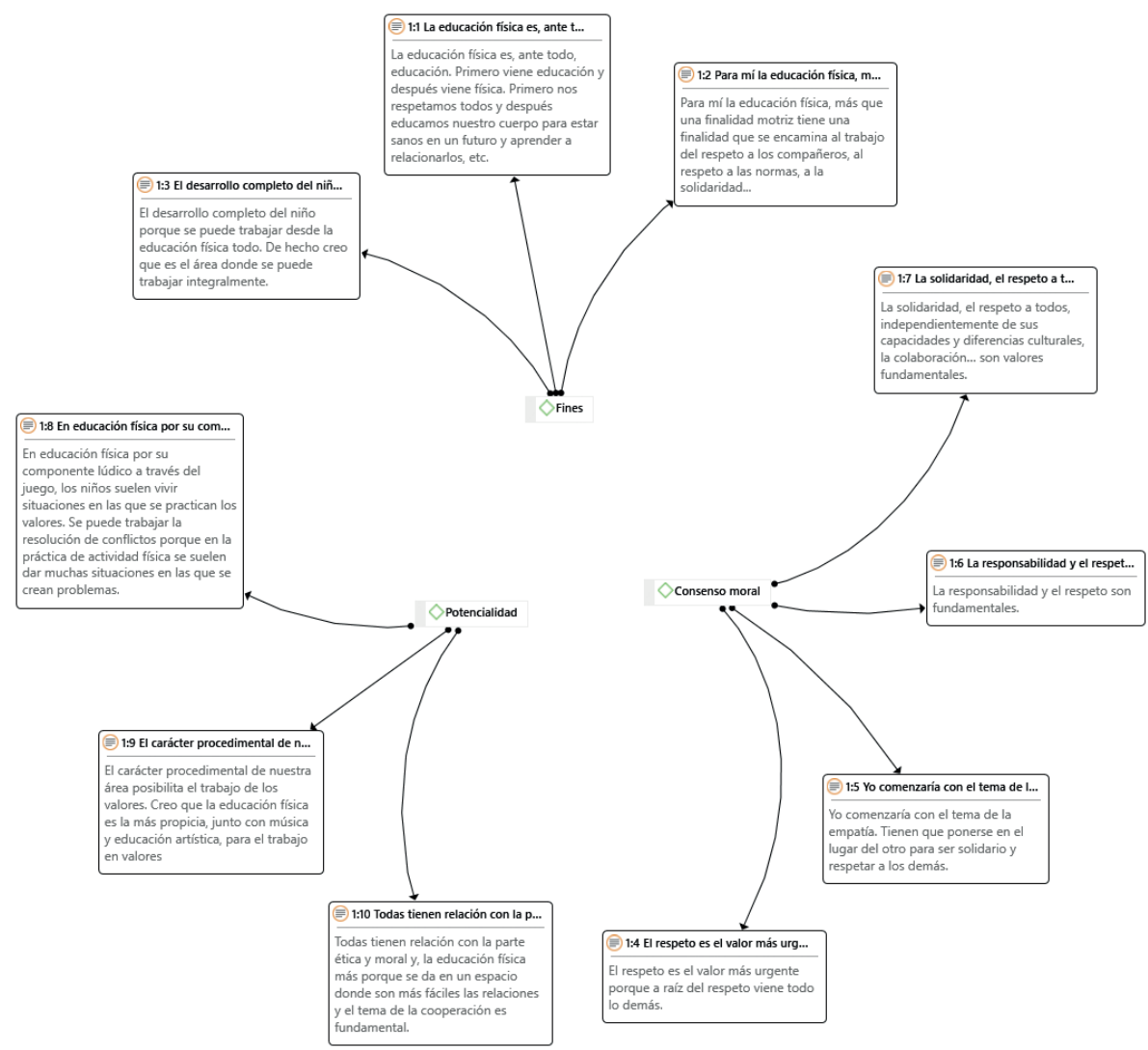

Figura 1. Red semántica de las categorías fines, consenso ético y potencialidad del área

En la figura 2 se muestra una red semántica con las categorías currículum, consenso explícito a nivel de centro y desarrollo curricular. La primera de ellas hace alusión a la existencia o no de los valores en el currículum del área según la percepción del profesorado. Los entrevistados manifiestan que en el nuevo currículum está presente el componente ético, pero añaden que los estándares de aprendizaje son muy ambiguos y difícilmente evaluables.

En cuanto a la categoría consenso explícito a nivel de centro, el profesorado manifiesta que los planes y proyectos que se desarrollan en sus respectivos centros están más enfocados en conseguir resultados de tipo cognitivo que resultados más enfocados al componente ético. Así mismo, argumentan que los planes se centran más en concretar medidas 
correctivas que en plasmar aquel trabajo preventivo y transversal que requiere el desarrollo de los valores. Con todo, manifiestan que existe un tratamiento de los valores en los proyectos y programas de los centros, aunque sostienen que su materialización es difícil si no existe una apuesta decidida por parte de los centros.

Con relación a la categoría desarrollo curricular, el profesorado de Educación Física percibe que los valores están presentes en sus diseños curriculares pero que existe mucha distancia entre lo que se planifica y lo que se lleva a la práctica. Sus creencias apuntan a que el trabajo de los valores depende más de las intenciones pedagógicas del profesorado que de la propia programación.

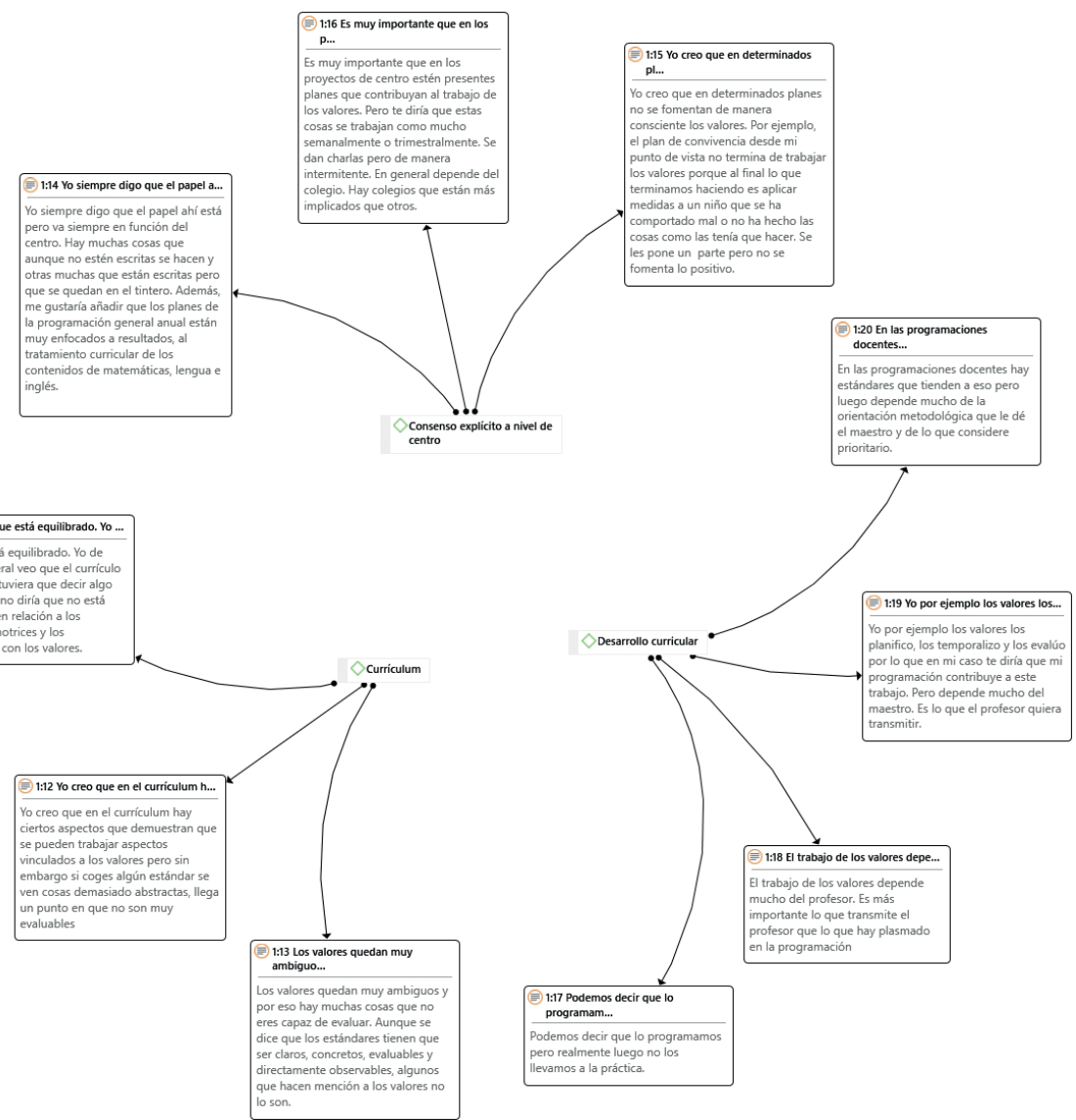

Figura 2. Red semántica de las categorías currículum, consenso explícito a nivel de centro y desarrollo curricular 
En la figura 3 se muestra la red semántica relativa a la categoría práctica docente. En relación a este aspecto, los profesionales entrevistados manifiestan que para generar un clima propenso para la educación en valores, el profesorado ha de ser una persona empática, respetuosa, accesible, comprometida, comprensiva y tolerante con el alumnado. Así mismo, coinciden en la necesidad de generar en el aula un contexto en el que el diálogo, la escucha y la reflexión estén presentes, y expresan, de manera contundente, que el/la profesor/a ha de ser un modelo y generar momentos donde la vivencia y experiencia del valor estén presentes.

En relación con la formación de los grupos, existe un acuerdo en cuanto a la consideración de la necesidad de hacer agrupamientos heterogéneos puesto que es en la diversidad y en la diferencia donde se aprenden y practican los valores. En este sentido, manifiestan que para la formación de los grupos hay que atender a determinados criterios tales como el género y las capacidades de carácter motriz, social y cognitivo.

En cuanto a los recursos materiales, los profesionales participantes expresan que éstos no son determinantes para la educación en valores. Según la opinión de los entrevistados lo realmente determinante es el enfoque que el profesorado de a sus clases de Educación Física, así como el compromiso que adquiera con este tipo de trabajo. Sin embargo, en relación con los recursos espaciales, argumentan que para trabajar valores relacionados con el respecto al medio ambiente, el entorno en el que se ubique la escuela y las salidas al entorno natural son elementos fundamentales. 
Creencias del profesorado de Educación Física en Educación Primaria sobre la educación en valores

Marta Gutiérrez Sánchez, Eduardo Romero Sánchez y Tomás IzQuierdo Rus

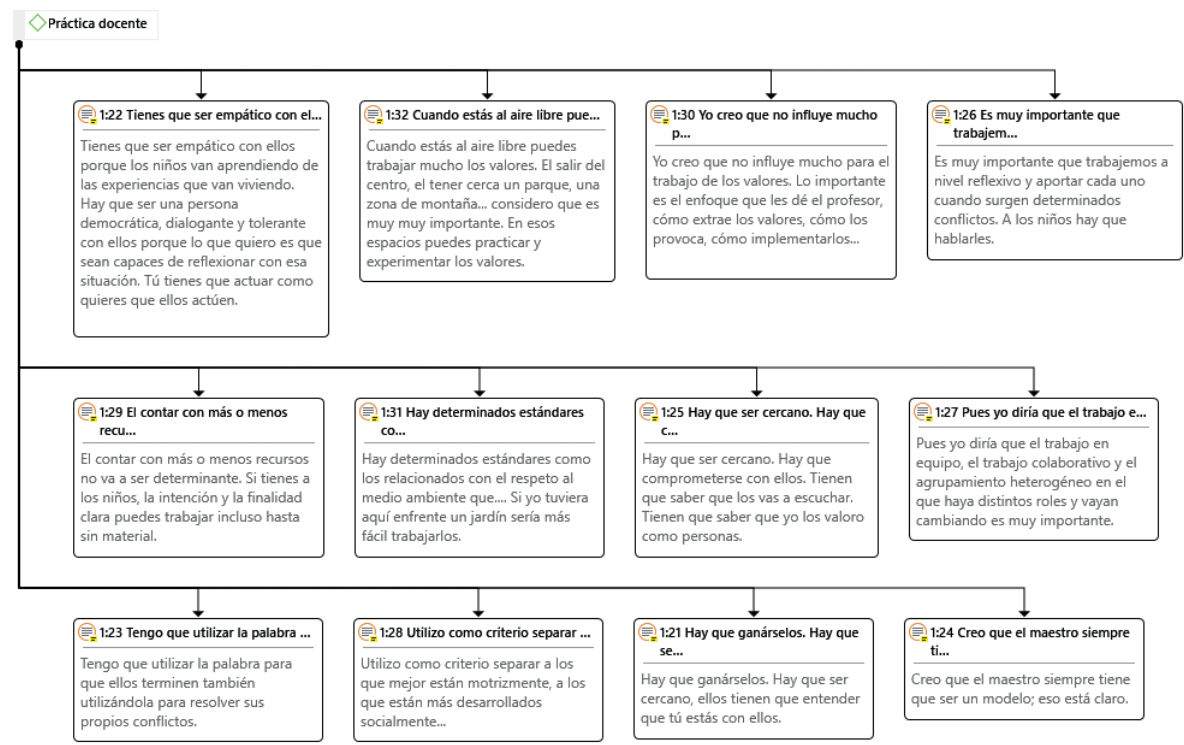

Figura 3. Red semántica de la categoría práctica docente

En la figura 4 se muestra la red semántica relativa a la categoría clima ético del centro. En este sentido los entrevistados manifiestan la importancia de la coordinación del profesorado, la necesidad de que existan acuerdos en torno a los valores que se han de trabajar y transmitir en el centro, y la pertinencia de que exista un clima relacional positivo para la adquisición de valores por parte del alumnado. En cuanto a las relaciones con las familias, los entrevistados insisten en la necesidad de colaboración constante entre la escuela y la familia puesto que ha de existir coherencia entre lo que se transmite en ambos espacios de socialización. Sin embargo, los profesionales perciben que existe muy poca coordinación entre el profesorado para tratar aspectos vinculados a la educación en valores, puesto que se le concede mayor importancia a aspectos vinculados al rendimiento académico en materias instrumentales. La coordinación en este sentido se circunscribe tan solo a actividades puntuales, lo que dificulta que exista una continuidad en el trabajo de los valores. Así mismo, y a pesar de la importancia que conceden a las relaciones de colaboración con las familias, consideran que la participación de estas es muy escasa y que existe incoherencia entre los valores que éstos transmiten y los que se tratan de inculcar en la escuela. 
Creencias del profesorado de Educación Física en Educación Primaria sobre la educación en valores Marta Gutiérrez Sánchez, Eduardo Romero Sánchez y Tomás IzQuierdo Rus

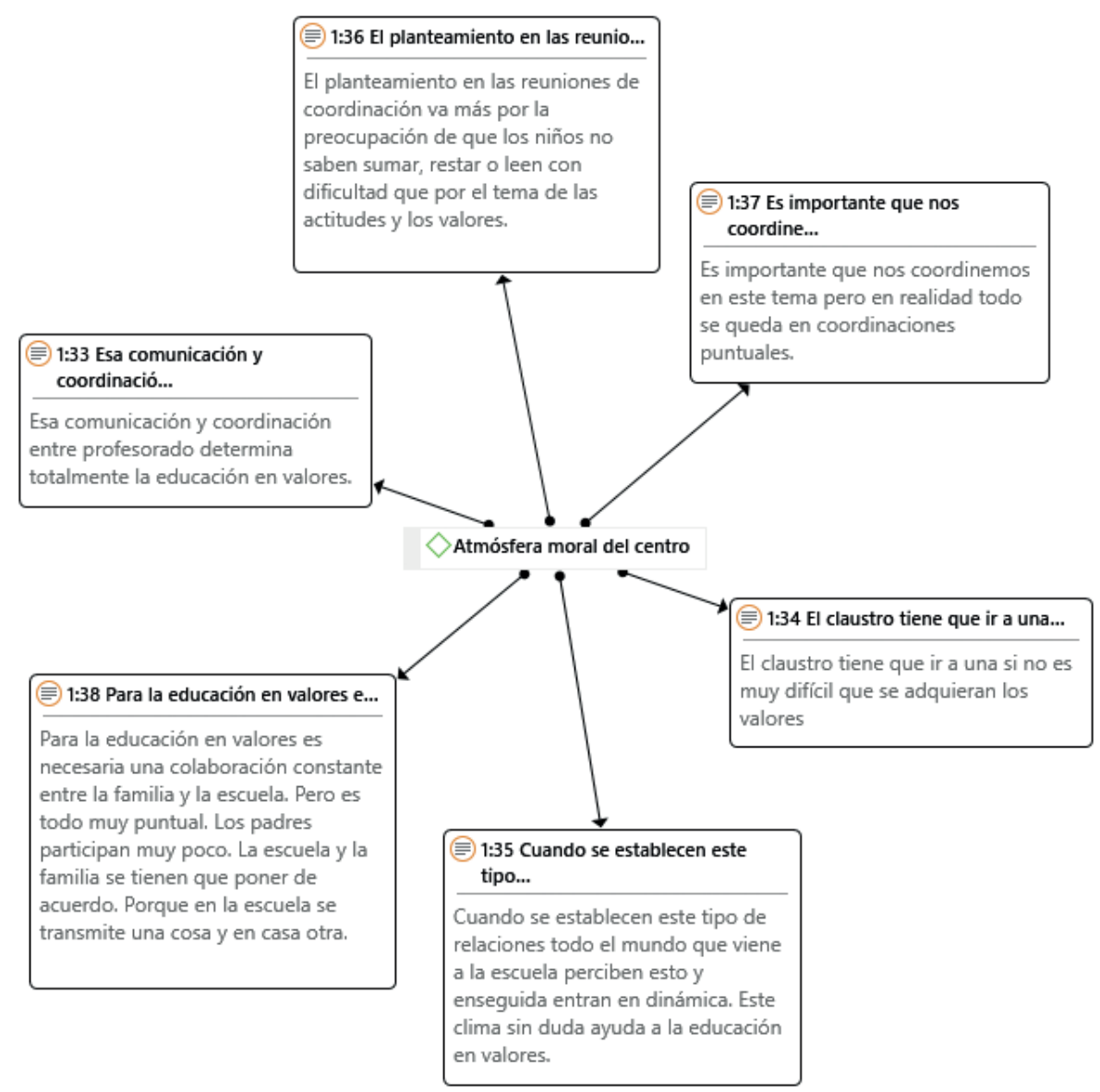

Figura 4. Red semántica de la categoría clima ético del centro

Por último, en la figura 5 se muestran los problemas que perciben los entrevistados para educar en valores. Destacan carencias en la formación inicial y continua en torno al componente axiológico de la educación, el valor que tanto la sociedad como las familias le otorgan a los resultados en las áreas instrumentales por encima de los valores, la escasa colaboración y formación de las familias, y el individualismo y la competitividad presentes en la sociedad actual.

Por otro lado, señalan que existen cuestiones del sistema educativo que se han de considerar para avanzar hacia un modelo en el que estén presentes los valores. En este sentido, consideran que existe un predominio de contenidos disciplinares en el currículum, que el sistema 
educativo se centra en valorar resultados de carácter instrumental relegando a un segundo plano las actitudes y valores, y que los continuos cambios legislativos dificultan que exista una línea de trabajo coherente, continua y permanente para trabajar los valores. Así mismo, subrayan la situación de interinidad de las plantillas docentes de los centros como un obstáculo para generar un plan de trabajo sostenible en el tiempo y compartido por todo el equipo docente.

La metodología que impera en nuestro actual sistema educativo es otro de los factores que se señala como problemático para avanzar hacia la educación en valores. El profesorado entrevistado considera que la vivencia de experiencias y enfoques integradores que aglutinen los contenidos de las distintas áreas son fundamentales para la adquisición de actitudes y valores por parte del alumnado. Así mismo, la falta de vocación del profesorado y las características de las pruebas de acceso a la profesión, en las que solo se miden conocimientos didáctico-disciplinares, se consideran como uno de los principales obstáculos para integrar los valores en nuestro sistema educativo. 
Creencias del profesorado de Educación Física en Educación Primaria sobre la educación en valores Marta Gutiérrez Sánchez, Eduardo Romero Sánchez y Tomás IzQuierdo Rus






\section{Discusión y conclusiones}

La cuestión de los valores merece especial atención en nuestro sistema educativo si queremos atender las exigencias de la sociedad actual. El profesorado constituye una pieza clave para avanzar hacia un sistema educativo que integre el componente axiológico de manera decidida, pero hemos de asumir que éste no actúa tan solo desde el marco de sus competencias profesionales sino también desde sus creencias sobre la enseñanza (Nespor, 1987). Por tanto, el conocimiento de las mismas nos ayuda a vislumbrar aquellos factores en los que hemos de incidir para que la práctica docente redunde en el desarrollo integral del alumnado.

Los resultados obtenidos constituyen la base para llevar a cabo acciones formativas, así como para identificar aquellos obstáculos que imposibilitan una intervención educativa que esté atenta al desarrollo del alumnado en todas sus dimensiones. Por tanto, las aportaciones de este estudio no son solo de carácter investigador sino también práctico. Nos ayudan a reflexionar sobre las cuestiones en las que hemos de avanzar para integrar el componente ético en nuestro sistema educativo.

En cuanto a la finalidad de la Educación Física en Educación Primaria, el profesorado expresa que el área contribuye al desarrollo integral del alumnado, cuestión compartida por Ruíz y Cabrera (2004) quienes han manifestado que los valores, como en cualquier otra área, son parte constitutiva de la Educación Física. Aluden también, como es obvio, a otras finalidades del área que tienen que ver con el desarrollo de la competencia motriz y la adquisición de competencias relacionadas con la salud en clara sintonía con lo que establece el Real Decreto 126/201, de 28 de febrero, por el que se establece el currículo básico de la Educación Primaria.

Por otro lado, con relación a la existencia de un consenso en torno a qué valores hemos de trabajar en Educación Primaria, se encuentran hallazgos muy parecidos en un estudio sobre creencias docentes sobre los determinantes de la actividad física (Álvarez, Velázquez-Buendía, Martínez-Gorroño y Díaz del Cueto, 2010). En este estudio se constata que los profesionales del área consideran una prioridad el fomento de valores sociales, tales como la tolerancia, la cooperación y el respeto, por encima de otros objetivos vinculados tradicionalmente al área de Educación Física.

Otro de los resultados de este estudio apunta que el área de Educa- 
ción Física posibilita de manera decidida que tenga lugar la educación en valores, cuestión ya señalada en la literatura especializada en el contexto nacional (Gutiérrez, 2003; Moncada, 2005) y en el internacional (Hellison, 2011). Los profesionales entrevistados, en clara sintonía con lo demostrado por Escartí, Pascual y Gutiérrez (2005), argumentan que las clases de Educación Física constituyen un lugar privilegiado para educar en valores puesto que las situaciones que se generan no se dan en otros contextos de aprendizaje.

En cuanto a la presencia de los valores en el currículum de Educación Física en la etapa de Educación Primaria, el profesorado concibe que está presente la dimensión ética en el nuevo diseño curricular, cuestión que merece especial consideración si tenemos en cuenta su importancia para la educación en valores, tal y como se ha argumentado en la literatura especializada (Gómez, 2003; Gutiérrez, 2003; Pavesio y Rivera, 2001). Sin embargo, manifiestan que los estándares de aprendizaje son muy abstractos o ambiguos y difíciles de evaluar, cuestión que debe ayudar a reflexionar sobre el planteamiento actual del currículum, si tenemos en cuenta que el Real Decreto 126/2014 insiste en que los estándares de aprendizaje "deben ser observables, medibles y evaluables (...) y que su diseño debe contribuir y facilitar el diseño de pruebas estandarizadas y comparables". (p.4)

Las creencias del profesorado en torno a la presencia de los valores en los proyectos que reflejan las intenciones educativas de la institución escolar, no son muy positivas si tenemos en cuenta la importancia que adquiere una visión compartida, plasmada en los documentos institucionales, en torno a los valores, para asegurar el compromiso de todo el centro escolar (Prat, 2001; Prat y Soler, 2003). Tampoco lo son las relativas al desarrollo curricular si tenemos en cuenta la importancia de planificar e incluir los valores en las programaciones docentes para que tenga lugar el desarrollo ético de los escolares (Sánchez, 2002; Venero, 2007).

Otra de las variables a tener en cuenta para la educación en valores está en relación con la práctica docente. Ya se argumentó que las relaciones que el profesorado establece con el alumnado, el uso de espacios y materiales y la formación de los grupos eran fundamentales para el trabajo de los valores (Gómez Rijo, 2005; Prat, 2001; Prat y Soler, 2003). Los hallazgos de este estudio coinciden con lo expresado con otros autores (Ortega y Mínguez, 2001; Touriñán, 2006) ya que los entrevistados consideran que para educar en valores el profesorado ha de ser un mo- 
delo y generar momentos donde la vivencia y experiencia del valor estén presentes.

Por último, el profesorado entrevistado identifica algunas cuestiones que obstaculizan que tenga lugar la educación en valores, coincidiendo en muchos casos con lo que muestra la literatura especializada. Así, Santos Guerra (2010) ha destacado problemas relativos a la formación inicial y continua del profesorado y Martínez, Esteban y Buxarrais (2010) han aludido a la escasa formación de las familias.

Este estudio, tras la revisión de la literatura, constituye una primera aproximación para conocer las creencias del profesorado de Educación Física en Educación Primaria sobre los factores que posibilitan la educación en valores en la Región de Murcia. Como principales limitaciones del estudio cabe destacar las siguientes: la investigación desarrollada en la temática es prácticamente inexistente y los estudios se centran más en sentar las bases teóricas para educar en valores que en recoger evidencias empíricas acerca de la temática de esta investigación; la escasa disponibilidad de los participantes puesto que la mayoría de ellos ocupan la mayor parte de su horario laboral en docencia directa o bien realizando otro tipo de tareas como las vinculadas a responsabilidades de centro, a la coordinación docente, a labores de acción tutorial o a la preparación de actividades de centro y de aula. Por tanto, sería conveniente realizar nuevas investigaciones con profesorado de Educación Física en Educación Secundaria Obligatoria y Bachillerato y con profesores/as de Educación Primaria especialistas en otras áreas para conocer si las creencias del profesorado difieren según especialidad y según etapa educativa. Así mismo, seria idóneo llevar a cabo estudios en esta línea que se propongan analizar las diferencias existentes entre el profesorado en ejercicio y el profesorado en formación.

Los resultados obtenidos podrían constituir el punto de partida para establecer medidas que posibiliten avanzar hacia un sistema educativo que integre y operativice la educación en valores. Conocer las creencias del profesorado nos permite diagnosticar una realidad y erigir algunas propuestas concretas en torno a las dimensiones estudiadas. La revisión y concreción de los valores en el diseño curricular, la formación del profesorado en metodologías que propicien la experiencia y vivencia del valor por parte del alumnado, el establecimiento de medidas en la gestión de los centros para posibilitar un proyecto de centro coherente y compartido por todo el profesorado, y la implementación de medidas 
que aseguren la colaboración de las familias con la escuela constituyen algunas de esas propuestas. Otra muy esperada por los y las que trabajamos en las distintas etapas del sistema educativo, tiene que ver con la necesidad de fijar unas líneas curriculares, metodológicas y axiológicas que perduren a lo largo del tiempo en la legislación educativa de nuestro contexto.

\section{Referencias}

Agúndez. D. (2015). Educar en valores. Teoría y práctica. Supervisión, Revista de Educación e Inspección, 37, 1-36.

Altarejos, F. y Naval, C. (2005) La educación cívica en una sociedad globalizada. Revista Galega do Ensino, 46, 841-862.

Álvarez, J.L., Velázquez-Buendía, R., Martínez-Gorroño, E. y Díaz del Cueto, M. (2010). Creencias y perspectivas docentes sobre objetivos curriculares y factores determinantes de actividad física. Revista Internacional de Medicina y Ciencias de la Actividad Física y del Deporte, 10 (38), 336-355.

Arufe, V. (2011). La educación en valores en el aula de educación física. ¿Mito o realidad? EmásF. Revista Digital de Educación Física, 9, 32-42,

Blumer, H. (1982). El interaccionismo simbólico: Perspectivas y método. Barcelona: Hora.

Bolívar, A. (1998). Educar en valores. Una educación en la ciudadanía. Sevilla: Consejería de Educación y Ciencia.

Bolívar, A. (2007). Dos valores á cidadanía: cambios e problemas persistentes. Eduga: Revista Galega do Ensino, 51, 26-29

Buendía, L., Colás, P., y Hernández, F. (1998). Métodos de investigación en psicopedagogía. Madrid: McGraw-Hill.

Colás, P. Buendía, L. y Hernández F. (2009). Competencias científicas para la realización de una tesis doctoral. Barcelona: Davinci.

Escartí, A., Pascual, C. y Gutiérrez, M. (2005). Responsabilidad Personal y Social a través de la educación física y el deporte. Barcelona: Graó.

Evans, J. (2001). La investigación en la educación física: en busca de comprensión y calidad en la enseñanza. En J. Devís (Coord.), La Educación Física, el deporte y la salud en el siglo XXI (pp. 111-126). Alcoy: Marfil.

Gómez Rijo, A. (2005). La enseñanza y el aprendizaje de los valores en la educación deportiva. Revista Internacional de Medicina y Ciencias de la Actividad Física y el Deporte, 5(18), 88-99.

Gómez Rodríguez, A. E. (2008). Educación para la ciudadanía. Una aproximación al estado de la cuestión. Enseñanza de las Ciencias Sociales, 7, 131-140.

Gómez, A. (2003). Educación Física y valores: análisis de la presencia de valores en el Currículo de Educación Física para la etapa de Primaria en la Comunidad Canaria. Actas del XXI Congreso Nacional de Educación Física El pensamiento del profesorado, Tenerife, España. 
Creencias del profesorado de Educación Física en Educación Primaria sobre la educación en valores

Marta Gutiérrez Sánchez, Eduardo Romero Sánchez y Tomás Izquierdo Rus

Gracia, J. y Gozálvez, V. (2016). Justificación filosófica de la educación en valores éticos y cívicos en la educación formal. Análisis crítico de la LOMCE. Teoría de la Educación, 28 (1): 83-103.

Gutiérrez Sanmartín, M. (2004). El valor del deporte en la educación integral del ser humano. Revista de Educación, 335, 105-126.

Gutiérrez, M. (2003). Manual sobre valores en la educación física y el deporte. Barcelona: Paidós.

Hellison, D.R. (2011). Teaching responsibility through physical activity. Champaign, IL: Human Kinetics.

Hernández-Álvarez, J.L, Velázquez-Buendía, R., Martínez-Gorroño, Ma. E. y Díaz del Cueto, M. (2010). Creencias y perspectivas docentes sobre objetivos curriculares y factores determinantes de actividad física, Revista Internacional de Medicina y Ciencias de la Actividad Física y el Deporte, 10(38), 336-355.

Ley Orgánica 8/1985, de 3 de julio, reguladora del Derecho a la Educación. Boletín Oficial del Estado, 159, 4 de julio de 1985, pp. 21015-21022.

Ley Orgánica 1/1990, de 3 de octubre, de Ordenación General del Sistema Educativo. Boletín Oficial del Estado, 238, 4 de octubre de 1990, pp. 28927-28942.

Ley Orgánica 2/2006, de 3 de mayo, de Educación. Boletín Oficial del Estado, 106, 4 de mayo de 2006, pp. 17158-17207.

Ley Orgánica 8/2013, de 9 de diciembre, para la Mejora de la Calidad Educativa. Boletín Oficial del Estado, 295, 10 de diciembre de 2013, pp. 97858-97921.

Marín, Ma.M. (2013). La Educación Física a través de los valores y el bilingüismo. Emotion. Revista de Educación, Motricidad e Investigación, 1, 155-172.

Martínez, M., Esteban, F., Buxarrais, M. (2011). Escuela, profesorado y valores. Revista de Educación, número extraordinario, 95-113.

Moncada, J. (2005). Año internacional del deporte y la educación física. Revista Educación, 29(2), 235-249.

Nespor, J. (1987). The Role of Beliefs in the Practice of Teaching. Journal of Curriculum Studies, 19(4). 317-328.

Orden ECD/65/2015, de 21 de enero, por la que se describen las relaciones entre las competencias, los contenidos y los criterios de evaluación de la educación primaria, la educación secundaria obligatoria y el bachillerato. Boletín Oficial del Estado, 25, 29 de enero de 2015, pp. 6986-7003.

Ortega, P. y Mínguez, R. (2001). Los valores en la educación. Barcelona: Ariel.

Pascual, C., Escarti, A. Llopis, P. Gutiérrez, M. Marín, D. \& Wright, P.M. (2011). Implementation Fidelity of a Program Designed to Promote Personal and Social Responsibility Through Physical Education: a Comparative Case Study. Research Quarterly for Exercise and Sport. 82(3), 499-511.

Pavesio, M. y Rivera, E. (2001). La presencia de valores en el currículum andaluz para la educación física de primaria. En A. Díaz y E. Segarra, E. (coord.) Actas del II congreso internacional de educación física y diversidad. Murcia, España.

Peiró, S. (2013). Los valores en la educación. Modelos procedimientos y técnicas. Madrid, España: Dykinson. 
Pérez Serrano, G. (1990). La investigación-acción. Aplicaciones al campo social y educativo. Madrid: Dykinson.

Prat, M. (2001). Actitudes, valores y normas en educación física: Reflexiones problemáticas y propuestas para su integración en la escuela. Tándem: Didáctica de la Educación física, 2, 7-20.

Prat, M. y Soler, S. (2003). Actitudes y valores y normas en la educación física y el deporte. Reflexiones y propuestas didácticas. Barcelona: INDE publicaciones.

Prat, M., Font, R., Soler, S. y Calvo, J. (2004). Educación en valores, deporte y nuevas tecnologías. Apunts. Educació Física i Esports, 78. 83-90.

Raventós, F. y Prats, E. (2012). Sociedad del conocimiento y globalización. Nuevos retos para la educación comparada. Revista Española de Educación Comparada, 20, 1940.

Real Decreto 126/2014, de 28 de febrero, por el que se establece el currículo básico de la Educación Primaria. Boletín Oficial del Estado, 25, 1 de marzo de 2014, pp. 19349-19420.

Reboul, O. (2009) Filosofía de la Educación. Barcelona: Da Vinci

Romero, E. y Gutiérrez, M. (2013). Posibilidades y amenazas para que las actuales sociedad de la información se conviertan en verdaderas sociedades del aprendizaje y del conocimiento. Revista Teoría de la Educación. Educación y Cultura en la Sociedad de la información, 14(3), 241-258.

Ruiz, C. (2010). La Educación en la sociedad postmoderna: Desafíos y oportunidades. Revista Complutense de Educación, 21 (1), 173-188.

Ruiz, G. y Cabrera, D. (2004). Los valores en el deporte. Revista de Educación, 335, 9-19.

Sánchez, J. (2002). La educación psicomotriz en el contexto de las ciencias de la educación. En M. Llorca, V. Ramos, J. Sánchez y A. Vega (Coords.), La práctica psicomotriz: Una propuesta educativa mediante el cuerpo y el movimiento (pp. 97-142). Málaga, España: Aljibe.

Santos Guerra, M.A. (2010). Una pretensión problemática: educar para los valores y preparar para la vida. Revista de Educación, 351, 23-47.

Touriñán, J.M. (2006). Educación en valores y experiencia axiológica: el sentido patrimonial de la educación, Revista Española de Pedagogía, 234(64), 227-247.

Venero, J.P. (2007). La clase de Educación Física como motor de cambio social. Reflexionando sobre actividades en la naturaleza, currículum oculto y valores. Retos. Nuevas Tendencias en Educación Física, Deporte y Recreación, 11, 51-53.

Wright, P.M., \& Burton, S. (2008). Implementation and Outcomes of a Responsibility - Based Physical Activity Program Integrated Into an Intact High School Physical Education Class. Journal of Teaching in Physical Education, 27, 138-154. 
